\title{
Duodenal plasmacytoma: a rare extramedullary localization simulating carcinoma of the head of the
} pancreas

\author{
G. Schoretsanitis, J.I. Livingstone, J.N. El-Japour ${ }^{1}$, N. Watkins and \\ C. Wastell
}

Academic Surgical Unit and ${ }^{1}$ Department of Histopathology, Chelsea \& Westminster Hospital, 369 Fulham Road, London SW10 9NH, UK

Summary: Plasmacytomas occurring in extramedullary sites are rare tumours, particularly so when located in the gastrointestinal tract. We report the case of a solitary extramedullary plasmacytoma arising in the duodenum and simulating a carcinoma of the head of the pancreas. Diagnostic and treatment options are discussed.

\section{Introduction}

Solitary extramedullary plasmacytoma (EMP) is a rare tumour, usually found in the upper respiratory tract or oral cavity but occasionally seen in the spleen, lymph nodes, lungs, liver, genital organs, kidneys or thyroid. ${ }^{1,2}$ Solitary EMP located in the duodenum is extremely rare, only five cases having been reported previously. ${ }^{3-7}$

We report here a case of solitary EMP of the duodenum which presented important diagnostic and therapeutic problems.

\section{Case report}

A 61 year old man presented with a 4 month history of flatulent dyspepsia, vomiting and epigastric discomfort after meals, constipation and weight loss. On examination, he was thin but without palpable abdominal abnormality or jaundice. Plain abdominal X-ray revealed food debris and gas in the stomach, whilst peripheral blood testing showed haemoglobin $11.4 \mathrm{~g} / \mathrm{dl}$, white blood cells $3.7 \times 10^{9} / 1$, an obstructive pattern of liver function but clotting within normal limits.

Barium meal showed narrowing and mucosal involvement of the second and third part of the duodenum associated with obstruction. Ultrasound scanning showed dilatation of the common bile duct with a mass in the head of the pancreas and ascites, confirmed by computer tomographic (CT) scanning which also showed the body and tail of the gland to be normal. There was no evidence of nodal or hepatic involvement.

Correspondence: J.I. Livingstone, F.R.C.S.

Accepted: 15 October 1993
At laparotomy, a mobile tumour was found in the head of the pancreas, eroding into the second and third parts of the duodenum. A radical pancreaticoduodenectomy (Whipple's procedure) was performed.

Histological examination revealed diffuse thickening of up to $2 \mathrm{~cm}$ depth over $6 \mathrm{~cm}$ of the duodenal wall with infiltration into the head of the pancreas, omental fat and distal common bile duct. Microscopically, the tumour consisted almost entirely of mature and immature plasma cells with a smaller component of lymphoplasmacytoid and lymphoid cells. Many of the tumour cells showed intranuclear (Dutcher bodies) or cytoplasmic (Russell bodies) immunoglobulin inclusions, which were predominantly IgM-type, with a smaller component of IgA and IgG. Limited kappa light chain restriction was found. The features of the tumour were those of a low-grade diffuse B-cell lymphoma of plasmacytoma type (Figure 1). There was no evidence of coeliac disease and both stomach and jejunum were unremarkable, the pancreas showing chronic inflammation, as did the gallbladder. The omental lymph nodes showed granulomatous reactive changes only.

Postoperative chest, abdominal and pelvic CT scan revealed no evidence of disease, and bone scanning was normal. Serum electrophoresis showed no paraproteins but slightly raised IgA. Urine electrophoresis showed two small Bence Jones kappa bands. Bone marrow trephine was within normal limits.

The patient was discharged on the 15 th postoperative day and remains well after 18 months of follow-up, without evidence of recurrent disease. Protein studies remain unchanged. 


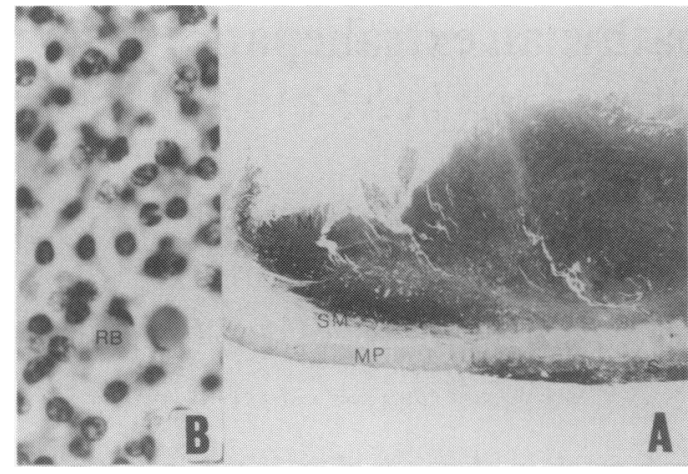

Figure 1 (A) The plasmacytoma infiltrated the full thickness of the wall of the duodenum. Mucosa (M), submucosa (SM), muscularis propria (MP) and serosa (S) muscularis mucosae (MM) (H \& E, original magnification $\times 6$ ). (B) The tr.mour cells were composed of mature and immature plasma cells with typical 'cart-wheel' nuclear chromatin pattern. Some cells contained cytoplasmic immunoglobulins (Russell bodies, RB) (PAS, original magnification $\times 1,200)$.

\section{Discussion}

Plasmacytomas are rare tumours, consisting of diffuse proliferation of plasma cells, and are classified into four groups: (1) multiple myeloma; (2) solitary myeloma of bone; (3) plasma cell leukaemia; and (4) extramedullary plasmacytoma. ${ }^{1}$ The interrelationship of these conditions is still unknown.

There are two types of EMP. The more common type represents a solitary extramedullary manifestation of multiple myeloma. Whether these lesions represent a primary isolated tumour or metastatic disease from a primary tumour in bone is unknown, because multifocal presentation of EMP

\section{References}

1. Dolin, S. \& Dewar, P. Extramedullary plasmacytoma. Am J Pathol 1956, 32: 83-103.

2. Hellweg, C.A. Extramedullary plasma cell tumours as observed in various locations. Arch Pathol 1943, 36: 95-111.

3. Hufferman, A. Plasmacytoma of pancreas and duodenum causing acute intestinal obstruction. Lancet 1947, i: 910

4. Derechin, M.M. Extraosseous plasmacytomas causing extrahepatic cholestasis and cardiac tamponade. Scand J Haemtol 1970, 7: 318 .

5. Pentimone, F., Camici, M., Cini, G. \& Levorato, D. Duodenal plasmacytoma: a rare extramedullary localisation simulating a carcinoma. Acta Haemat 1979, 61: 155-160.

6. Hirakawa, T., Fukuda, Y., Tabata, M. et al. Primary duodenal plasmacytoma: an autopsy case. Jap J Clin Med 1978, 36: 3278-3279.

7. Mannell, A. Primary isolated extramedullary plasmacytoma of the duodenum. Aust NZ J Surg 1979, 49: 577-580.

8. Sharma, K.D. \& Shrivastav, J.D. Extramedullary plasmacytoma of gastrointestinal tract: case report of a plasmacytoma of the rectum and a review of the literature. Arch Pathol 1961, 71: 229-233. has been reported not only in the upper air passages, but also in the gastrointestinal tract. ${ }^{8}$ The second, very rare, type of EMP is characterized by an absence of bone lesions, Bence Jones proteinuria and bone marrow involvement. However, the typical manifestations of multiple myeloma may appear months or years after histological diagnosis of extramedullary localized disease. ${ }^{1,5}$

Gammopathy (the presence of abnormal paraproteins) makes the diagnosis of primary EMP uncertain, as it is thought that EMPs do not produce abnormal paraproteins. ${ }^{1}$ Other work, however, suggests that gammopathy may be consistent with EMP provided the abnormal immunoglobulin disappears after resection of the tumour. ${ }^{9}$

The gastrointestinal tract is an unusual site for EMP, the majority of those which do occur being located in the stomach or small bowel. ${ }^{10-14}$ Routine preoperative investigations are unlikely to suggest the diagnosis unless a tissue biopsy is obtained. Radiological features are those of a homogeneous mass and are relatively non-specific.

The prognosis of patients with EMP of the duodenum is uncertain as so few patients have been reported. Of those cases in the literature, one was treated by local excision succumbing 15 months later to multiple myeloma, ${ }^{5}$ one underwent radical pancreaticoduodenectomy and was well up to follow-up of only 3 months ${ }^{7}$ whilst one case was only discovered at post-mortem examination. ${ }^{6} \mathrm{We}$ performed a radical procedure, as there was no evidence of metastatic disease and our patient remains disease free 18 months postoperatively.

It is not possible to identify the ideal method of treatment of EMP. However, we agree that all patients should be followed-up for life with repeated bone marrow aspiration and protein studies to detect the development of multiple myeloma.

9. Wiltshaw, E. The natural history of extramedullary plasmacytoma and its relation to solitary myeloma of bone and myelomatosis. Medicine 1976, 55: 217-238.

10. Preud'Homme, J., Galian, A., Danon, F., Marti R. \& Ramboud, J. Extramedullary plasmacytoma with gastric and lymph node involvement: an immunological study. Cancer 1980, 46: 1753-1758.

11. Funakoshi, N., Kanoh, T., Kobayashi, Y., Miyake, T., Uchino, H. \& Ochi, K. IgM-producing gastric plasmacytoma. Cancer 1984, 54: 638-643.

12. Rygaard-Olsen, C., Boedker, A., Emus, H. \& Olsen, H Extramedullary plasmacytoma of the small intestine. Cancer 1982, 50: 573-576.

13. Gleason, T. \& Hammar, S. Plasmacytoma of the colon: case report with lambda chain, demonstrated by immunoperoxidase studies. Cancer 1982, 50: 130-133.

14. Bhatnagar, B. \& Khanna, S. Extramedullary plasmacytoma of the anal canal. J $R$ Coll Surg Edinb 1983, 28: 328-330. 